\title{
Twin migration in Fe-based bec crystals: theory and experiments
}

\author{
A. Ojha ${ }^{a}$, H. Sehitoglu ${ }^{\text {a*}}$, L. Patriarca ${ }^{a}$ and H.J. Maier ${ }^{b}$ \\ ${ }^{a}$ Department of Mechanical Science and Engineering, University of Illinois at \\ Urbana-Champaign, 1206 W. Green Street, Urbana, IL 61801, USA; ${ }^{b}$ Institut für Werkstoffkunde, \\ Leibniz Universität Hannover, An der Universität 2, D-30823 Garbsen, Germany
}

(Received 23 October 2013; accepted 14 February 2014)

\begin{abstract}
We establish an overall energy expression to determine the twin migration stress in bcc metals. Twin migration succeeds twin nucleation often after a load drop, and a model to establish twin migration stress is of paramount importance. We compute the planar fault energy barriers and determine the elastic energies of twinning dislocations including the role of residual dislocations $\left(b_{r}\right)$ and twin intersection types such as $\left\langle\begin{array}{lllll}1 & 1 & 0\rangle\end{array}\right\rangle,\left\langle\begin{array}{llll}1 & 1 & 3\end{array}\right\rangle$ and $\langle 210\rangle$. The energy expression derived provides the twin migration stress in relation to the twin nucleation stress with a ratio of $0.5-0.8$ depending on the resultant residual burgers vector and the intersection types. Utilizing digital image correlation, it was possible to differentiate the twin nucleation and twin advancement events experimentally, and transmission electron microscopy observations provided further support to the modelling efforts. Overall, the methodology developed provides an enhanced understanding of twin progression in bcc metals, and most importantly the proposed model does not rely on empirical constants. We utilize $\mathrm{Fe}-50 \mathrm{at} . \% \mathrm{Cr}$ in our experiments, and subsequently predict the twin migration stress for pure $\mathrm{Fe}$, and $\mathrm{Fe}-3 \mathrm{at} . \% \mathrm{~V}$ from the literature showing excellent agreement with experiments.
\end{abstract}

Keywords: molecular dynamics; TEM; twinning

\section{Introduction}

Twin migration refers to the movement of the twin boundary after twin nucleation often compounded by the interaction with other conjugate twins. It is well known that the determination of twin migration stresses remains a significant challenge in metals [1-3]. The twin migration behaviour has a marked influence on the flow behaviour of alloys subsequent to twin nucleation. An accurate determination of twin migration stresses must consider the mutual interaction of primary and conjugate twins and the resultant reactions at the twin interface. The present work is focused on developing a combined atomistic-continuum framework for bcc metals to determine the twin migration stress with special emphasis on the role of intersections. Experiments are conducted on single crystals to measure precisely the twin nucleation and twin migration stresses by monitoring the twin-induced strain evolution. The measurements of stresses are compared with the proposed theory with excellent agreement.

\footnotetext{
*Corresponding author. Email: huseyin@illinois.edu 
Previous works on bcc metals have discovered different reactions at the twin boundaries [4-6], and classified the twin-twin intersection types based on the common line of intersection of the interacting twins [7,8]. If $m$ and $n$ are the plane normals of the two interacting twins, the intersection type is the vector corresponding to their common intersection line, and is given by the cross product of $m$ and $n$. Levasseur [7] and Mahajan [8] have shown that five types of intersections can exist, namely $\left\langle\begin{array}{llll}1 & 1 & 0\end{array}\right\rangle$, $\langle 210\rangle,\langle 113\rangle,\left\langle\begin{array}{llll}1 & 1 & 1\end{array}\right\rangle$ and $\left\langle\begin{array}{lll}1 & 3 & 5\end{array}\right\rangle$ and the outcomes can be predicted based on the intersection type. Figure 1(a) and (b) shows two of the possible intersection types, $\left\langle\begin{array}{llll}1 & 1 & 3\end{array}\right\rangle$ and $\left\langle\begin{array}{lll}1 & 1 & 0\end{array}\right\rangle$ encountered in bcc crystals documented in the present study (refer to Table 1 for the designation of twin systems in Roman numerals). In addition to $\left\langle\begin{array}{llll}1 & 1 & 3\end{array}\right\rangle$ and $\left\langle\begin{array}{llll}1 & 1 & 0\end{array}\right.$, the proposed model in general can handle all intersection types (see Table 2) that produce varying magnitude of residual dislocations. The residual dislocation left at the twin boundary is the difference in the burgers vector of the incoming and the outgoing dislocation, and is given by $b_{r}=b_{1}-b_{2}$ where $b_{1}$ is the burgers vector of the incoming dislocation and $b_{2}$ that of the outgoing dislocation. The $b_{1}$ and $b_{2}$ lie in the matrix and the twin coordinates, respectively, and $b_{2}$ requires a coordinate transformation to calculate the $b_{r}$. The following scenario can develop when the incident twin encounters a twin in a conjugate system. The dislocation reaction can leave a residual burgers vector at the boundary, and a resultant dislocation glides along the twin interface. The term
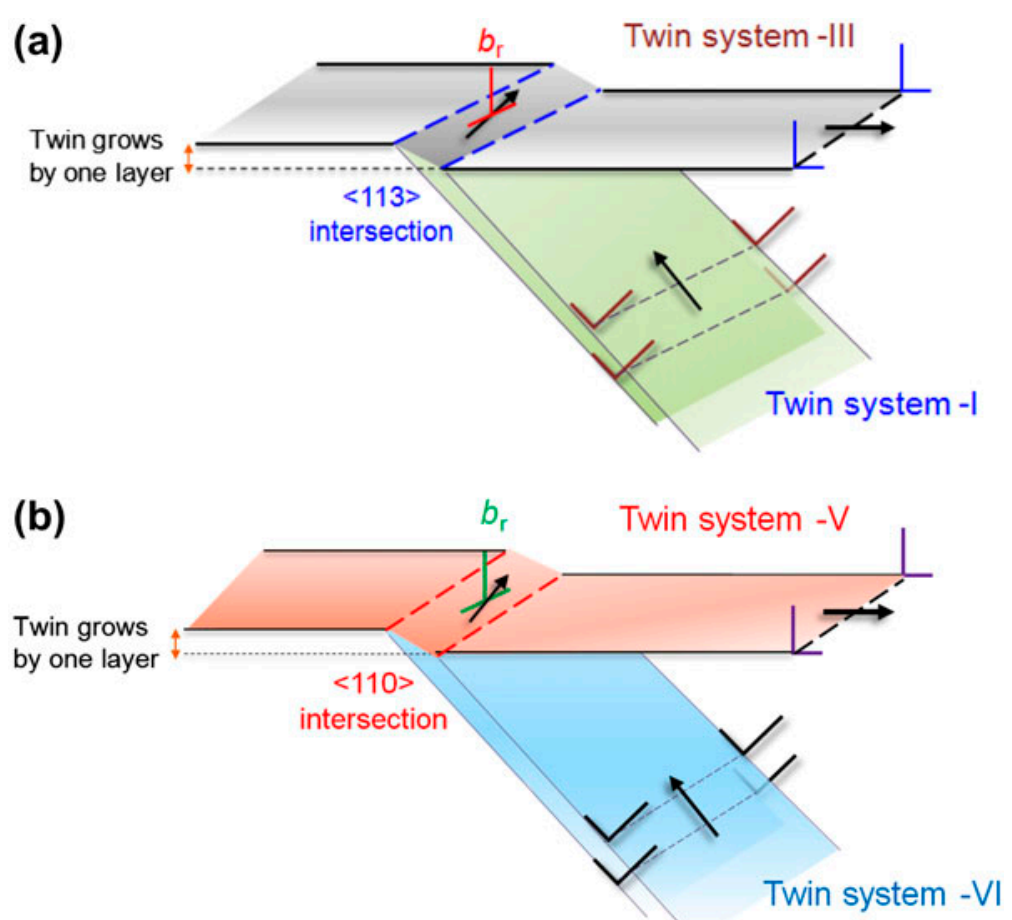

Figure 1. (colour online) (a) $\left\langle\begin{array}{llll}1 & 1 & 3\end{array}\right\rangle$ and (b) $\left\langle\begin{array}{llll}1 & 1 & 0 & 0\end{array}\right.$ intersection types encountered in a bcc crystal. The intersection type is based on the line of the intersection of the interacting twin systems. 
Table 1. (colour online) Designation of the possible activated twin systems when a bcc crystal is subjected to tension or compression in different arbitrary orientations. The colours are coordinated with the images.

\begin{tabular}{|c|c|c|c|c|c|c|}
\hline & $\bar{I}$ & II & III & IV & $\overline{\mathrm{V}}$ & VI \\
\hline $\begin{array}{l}\text { Twin } \\
\text { systems }\end{array}$ & {$\left[\begin{array}{lll}1 & 1 & 1\end{array}\right]\left(\begin{array}{lll}1 & \overline{2} & 1\end{array}\right)$} & {$\left[\begin{array}{lll}1 & \overline{1} & 1\end{array}\right]\left(\begin{array}{lll}1 & 2 & 1\end{array}\right)$} & 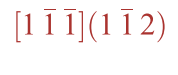 & {$\left[\begin{array}{lll}\overline{1} & \overline{1} & 1\end{array}\right]\left(\begin{array}{lll}2 & \overline{1} & 1\end{array}\right)$} & {$\left[\begin{array}{lll}\overline{1} & 1 & 1\end{array}\right]\left(\begin{array}{lll}1 & 2 & \overline{1}\end{array}\right)$} & {$\left[\begin{array}{lll}1 & 1 & \overline{1}\end{array}\right]\left(\begin{array}{lll}\overline{1} & 2 & \overline{1}\end{array}\right)$} \\
\hline
\end{tabular}

Table 2. (colour online) Pair of twin systems in bcc metals that interact to yield different types of intersections.

\begin{tabular}{|c|c|c|c|c|c|}
\hline Intersection & $\left\langle\begin{array}{lll}1 & 1 & 0\end{array}\right.$ & $\left\langle\begin{array}{lll}2 & 1 & 0\end{array}\right.$ & $\left\langle\begin{array}{lll}1 & 1 & 1\end{array}\right.$ & $\left\langle\begin{array}{lll}1 & 13 & 3\end{array}\right.$ & $\left\langle\begin{array}{llll}5 & 3 & 1\end{array}\right\rangle$ \\
\hline Twin systems & $\begin{array}{c}\text { I, II } \\
\text { V, VI }\end{array}$ & $\begin{array}{l}\text { I, V } \\
\text { I, VI } \\
\text { II, V } \\
\text { II, VI }\end{array}$ & $\begin{array}{l}\text { III, V } \\
\text { IV, VI }\end{array}$ & $\begin{array}{l}\text { I, III } \\
\text { I, IV } \\
\text { III, IV }\end{array}$ & $\begin{array}{c}\text { II, III } \\
\text { II, IV } \\
\text { III, VI } \\
\text { IV, V }\end{array}$ \\
\hline
\end{tabular}

'incorporation' or 'absorption' has been used to describe this encounter leading to the modified progression of twin boundaries. Upon twin-twin interaction, an $\frac{a}{6}\left\langle\begin{array}{lll}1 & 1 & 1\end{array}\right\rangle$ dislocation is formed as a result of the interaction glides along the twin boundary thus translating the boundary by one layer. The stress corresponding to twin boundary translation is referred to as the twin migration stress.

In this study, we choose single crystal orientations favourable for twinning, and the incoming and outgoing twin-slip systems are well defined. The slip systems that are activated will be discussed later in this section. We measure the crystal orientation of the samples and the orientation of the twinned domains such that three dimensional planes and directions are all indexed. Furthermore, by choosing Fe-50at.\%Cr crystals in our experiments, we obtain twinning at room temperature circumventing the need to cool to very low temperatures or apply high strains, and the associated issues with temperature, test control and accurate local strain measurements. We measure precisely the stress at which spontaneous twin nucleation occurs, the subsequent interaction of twin systems and the twin migration stress. The slip and twin systems are well known in Fe-50at.\%Cr crystals but the outcome of their interactions are not as well known, and we provide a deeper understanding of this characterization with the aid of simulation tools.

Atomistic simulations are undertaken to give further insight into the detailed mechanisms of the twin-twin interaction, particularly at the interfaces where the planar fault energies are modified because of the high internal stresses. We use molecular dynamics (MD) to study the interactions for the loading cases similar to the experimental loading orientations. We evaluate the step by step evolution of twin-twin interaction at an atomic level, thereby giving a detail picture of the twin migration process. We devised a special method of extracting the unstable twin energies from MD in the presence of other defects. Moreover, identifying the systems (planes and directions) of the interacting twins is easily accomplished by means of visualization tools. In this work, we use $\mathrm{MD}$ in conjunction with the experiments to identify the possible outcomes of the 
interacting twins through reactions, and to study the effect of the residual dislocation on the energetics of the twin migration process. We do so by incorporating the energy associated with the interacting twins and the residual dislocation, and the energy barriers that a dislocation should overcome in order to migrate the twin boundary. A number of experimental results have shown that the $b_{r}$ left at the twin boundary depends on the intersection type of the interacting twins $[7,8]$. In this work, we focus on three intersection types, namely $\left\langle\begin{array}{llllll}1 & 1 & 0\end{array},\left\langle\begin{array}{llll}2 & 1 & 0\end{array}\right\rangle\right.$ and $\left\langle\begin{array}{llll}1 & 1 & 3\end{array}\right\rangle$ and the possible outcomes of these intersections. The importance of $b_{r}$ is highlighted in the present study because it affects the local strain fields, and thus the twin migration stress in bcc metals. During the process of twin migration, the $\frac{a}{6}\left\langle\begin{array}{lll}1 & 1 & 1\end{array}\right\rangle$ dislocation has to overcome an energy barrier which is quantitatively represented by the generalized planar fault energy (GPFE). The details of the GPFE will be discussed later. The works of Ezaz et al. [9] on $\mathrm{Cu}$ and Sangid et al. [10] on $\mathrm{Ni}$ and $\mathrm{Cu}$ using atomistic calculations show that the presence of $b_{r}$ elevates the energy barrier required to nucleate a dislocation in different grain boundaries including the twin. Taking these findings in fcc metals into consideration, we explore the effects of $b_{r}$ on the twin migration process in bcc metals, and develop an energy-based formulation that quantifies the twin migration stress as a function of $b_{r}$.

The experiments in this study utilize single crystals of $\mathrm{Fe}-50 \mathrm{at} . \% \mathrm{Cr}$ oriented in $\left[\begin{array}{lll}0 & \overline{1} & 0\end{array}\right],\left[\begin{array}{lll}1 & 0 & 1\end{array}\right]$ and $\left[\begin{array}{lll}1 & \overline{1} & 1\end{array}\right]$ directions. It is well known that the activation of certain twinslip systems depends on the resolved shear stress on the slip-twin system, and can be calculated using the relation:

$$
\tau_{\mathrm{RSS}}=\sigma \cos (\lambda) \cdot \cos (\varphi)
$$

where $\lambda$ is the angle between the slip direction and the loading axis, $\varphi$ the angle between slip plane normal and the loading axis and $\sigma$ is the applied stress. The stereographic triangles in Figure 2 show the possible activated twin-slip based on the Schmid factor (SF). However, unlike slip which is bidirectional, there exists a unique shear direction for twinning. The red line in each of the stereographic triangles differentiates the regions where twinning is favourable over slip or vice versa. In our analysis, the $\left\langle\begin{array}{llll}1 & 1 & 1\end{array}\right\rangle\left\{\begin{array}{lll}1 & 1 & 2\end{array}\right\}$ twin systems having a SF larger than 0.35 is used as a criterion to define the regions (shaded area in Figure 2) where twinning is expected. The orientations in the present study are chosen such that in the case of $\left[\begin{array}{ll}0 & 1\end{array}\right]$ tension, there are four twin
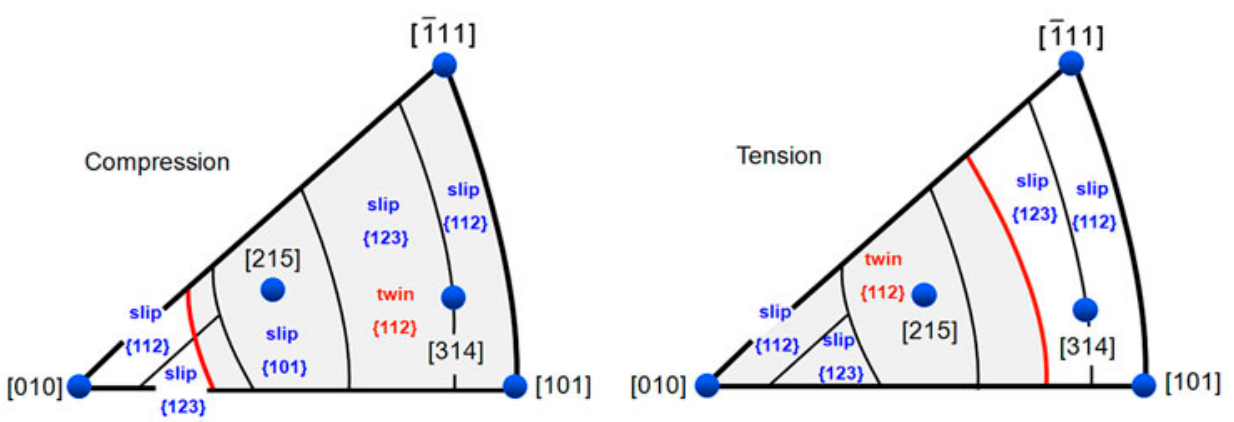

Figure 2. (colour online) Stereographic triangles showing different loading orientations in compression and tension, and the possible slip and twin systems. 
systems with high SF of 0.47 , in the case of [ $\left.\begin{array}{lll}1 & 1 & 1\end{array}\right]$ compressive orientation, there are three twin systems with SF of 0.31 and finally for the [llll 101 compression case, there are two twin systems with SF of 0.47 . Table 3 also reports the possible number of twins (n) that can be observed when the crystal is subjected to a specific loading orientation. Therefore, higher incidences of twin intersections are expected for the $\left[\begin{array}{lll}0 & 1 & 0\end{array}\right]$ tensile case while for the case of [ $\left[\begin{array}{lll}1 & 0 & 1\end{array}\right]$ compression, we would expect the lowest number of intersections. The $\left[\begin{array}{lll}1 & 1 & 1\end{array}\right]$ compression has the lowest hardening levels because it exhibits the lowest residual burgers vector pointing to the role of both the residual burgers vector and the number of twin systems. We predict the twin migration stresses accurately in all these cases without adjustable parameters.

\section{Experimental methods}

Fe-50at.\%Cr single crystals with loading axis parallel to [ $\left[\begin{array}{lll}1 & \overline{1} & 1\end{array}\right],\left[\begin{array}{lll}1 & 0 & 1\end{array}\right]$ and $\left[\begin{array}{lll}0 & \overline{1} & 0\end{array}\right]$ orientations were grown in a $\mathrm{He}$ atmosphere using the Bridgman technique. Electro-discharge machining was used to cut the samples into the dimensions $4 \mathrm{~mm} \times 4 \mathrm{~mm} \times 10$ $\mathrm{mm}$. Electron back scattering diffraction (EBSD) was used to determine the normal and transverse orientations of the samples. The samples were solution annealed at $900{ }^{\circ} \mathrm{C}$ for $1 \mathrm{~h}$ followed by a water quench prior to loading. For compressive tests, displacement controlled experiments were performed using a servo-hydraulic load frame. The tests were carried out at a strain rate of $5 \times 10^{-3} \mathrm{~s}^{-1}$ at room temperature. The nominal strain was measured using an extensometer. The digital image correlation (DIC) technique in combination with scanning electron microscopy enabled us to mark the interaction of twins and the evolution of twin migration. Ex situ high-resolution images $(0.44 \mu \mathrm{m} /$ pixel $)$ were obtained utilizing an optical microscope. The high-resolution images of the selected DIC area allow to capture a significant portion of the surface involved in the deformation, and with the adopted resolution, strain heterogeneities derived from twinning can be recognized. The high-resolution images enable the better characterization of the local strain magnitudes that are associated with twin-twin interaction regions. For higher resolution microstructural studies, transmission electron microscopy (TEM) was employed. For TEM work, $1 \mathrm{~mm}$ thick slices were cut normal to the loading direction of the crystal and then mechanically ground and polished down

Table 3. (colour online) Possible number of activated twin systems when a bcc crystal is subjected to tension or compression in different orientations. The maximum SF and the possible intersection types corresponding to the activated twins are also tabulated.

\begin{tabular}{|c|c|c|c|c|c|}
\hline & \multirow[b]{2}{*}{$\begin{array}{l}\text { Loading } \\
\text { orientation }\end{array}$} & \multicolumn{4}{|c|}{ Experimentally observed twin systems } \\
\hline & & $\begin{array}{c}\text { Planes and } \\
\text { directions }\end{array}$ & SF & $\begin{array}{l}\text { Number of } \\
\text { twin systems }\end{array}$ & $\begin{array}{c}\text { Possible } \\
\text { intersection types }\end{array}$ \\
\hline Compression & $\begin{array}{l}{\left[\begin{array}{lll}1 & 0 & 1\end{array}\right]} \\
{\left[\begin{array}{lll}1 & 1 & 1\end{array}\right]}\end{array}$ & $\begin{array}{l}\text { I, II } \\
\text { I, III } \\
\text { I, IV }\end{array}$ & $\begin{array}{l}0.47 \\
0.31 \\
0.31\end{array}$ & $\begin{array}{l}2 \\
3\end{array}$ & $\begin{array}{lll}\left\langle\begin{array}{lll}1 & 1 & 0\end{array}\right. \\
\left\langle\begin{array}{lll}1 & 1 & 3\end{array}\right. \\
\left\langle\begin{array}{lll}1 & 1 & 3\end{array}\right.\end{array}$ \\
\hline Tension & {$\left[\begin{array}{lll}0 & \overline{1} & 0\end{array}\right]$} & $\begin{array}{l}\text { II, V } \\
\text { I, VI }\end{array}$ & $\begin{array}{l}0.47 \\
0.47\end{array}$ & 4 & 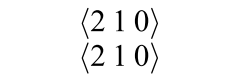 \\
\hline
\end{tabular}


to a foil thickness of $0.1 \mathrm{~mm}$. Large electron transparent areas were obtained by conventional twin-jet electropolishing with an electrolyte consisting of 5 pct perchloric acid in methanol. Electropolishing was done at a temperature of $-23{ }^{\circ} \mathrm{C}$ and a potential of 21 V. Whenever possible, two-beam imaging conditions were employed for imaging. Burgers vectors were determined based on the $g \cdot b=0$ invisibility criterion.

The present work is characterized by an extensive use of experimental strain measurements obtained via DIC methodology. Strain measurements from DIC and crystal orientations from EBSD were mainly used for studying the local strain fields associated with the deformation mechanism (twin-twin interactions). In particular, the application of this methodology for the selected single crystals allows identifying the points on the stress-strain curve where slip and twin nucleate, and follows the associated strain evolution. The main advantage of using DIC relates the possibility to quantify the local strain values associated with the deformation mechanisms. Typically, for bcc materials, twin nucleation can also be identified on the stress-strain curve when the load drops and is accompanied by an audible click. In some cases, the load drop can occur even in the elastic region of the stress-strain curve [11]. It is important to verify the presence of the twinned regions on the sample, since in some bcc materials, under particular conditions, slip nucleation can produce noticeable load drop as well. In all the cases in the present work, the crystal orientations displaying twinning have been successively analysed using EBSD.

Twin nucleation at a critical resolved shear stress $\left(\tau^{T}\right)$ is followed by twin migration at a CRSS of $\tau^{M}$ which is lower. Luca and co-workers [12] have successfully established the slip nucleation, twin nucleation and twin migration stresses for $\mathrm{Fe}-50 \mathrm{at} . \% \mathrm{Cr}$ using tensile and compressive tests for specifically oriented samples. In the present work, we study the strain field associated with the residual dislocation left at the twin boundary for an isolated twin-twin interaction, and quantify its effect on the twin migration stress $\tau^{M}$.

\section{Experimental results}

In order to understand the role of residual dislocations in modifying the outcomes of the twin-twin interaction process, and the effects on the twin migration stress, different types of twin-twin intersections are considered which are presented in Table 3. It is observed that different types of intersections yield different magnitudes of residual dislocations, and thus affect the outcomes of the interaction. Table 4 shows the activated twin systems in $\mathrm{Fe}-50 \mathrm{at} . \% \mathrm{Cr}$, their corresponding intersection types and the residual dislocations left at the twin boundary. The activated twin systems observed in all the cases correspond to the maximum SF. In the present work, the following three types of intersections, namely $\left\langle\begin{array}{llll}1 & 1 & 0\end{array}\right\rangle,\left\langle\begin{array}{llll}2 & 1 & 0\end{array}\right\rangle$ and $\left\langle\begin{array}{llll}3 & 1 & 1\end{array}\right\rangle$ are studied for which the magnitudes of the residual dislocations are different. In all the cases, the incident twin is blocked and the residual dislocations are left in the twin boundary. If we observe the magnitude of the residual dislocation in Table 3 , the $\left\langle\begin{array}{llll}1 & 1 & 0\end{array}\right\rangle$ intersection yields a higher value $\left(b_{r}=1.0 a\right)$ compared to the $\langle 113\rangle$ and $\langle 210\rangle$ cases $\left(b_{r}=0.82 a\right)$. Consequently, we would expect the twin migration stress to be higher for the $\langle 110\rangle$ intersection type compared to the $\left\langle\begin{array}{llll}1 & 1 & 3\end{array}\right\rangle$ and $\langle 210\rangle$ types.

The identification of the intersection types facilitates to predict the outcomes of the interactions. A prior knowledge of the SF for different twin systems in different loading 
A. Ojha et al.

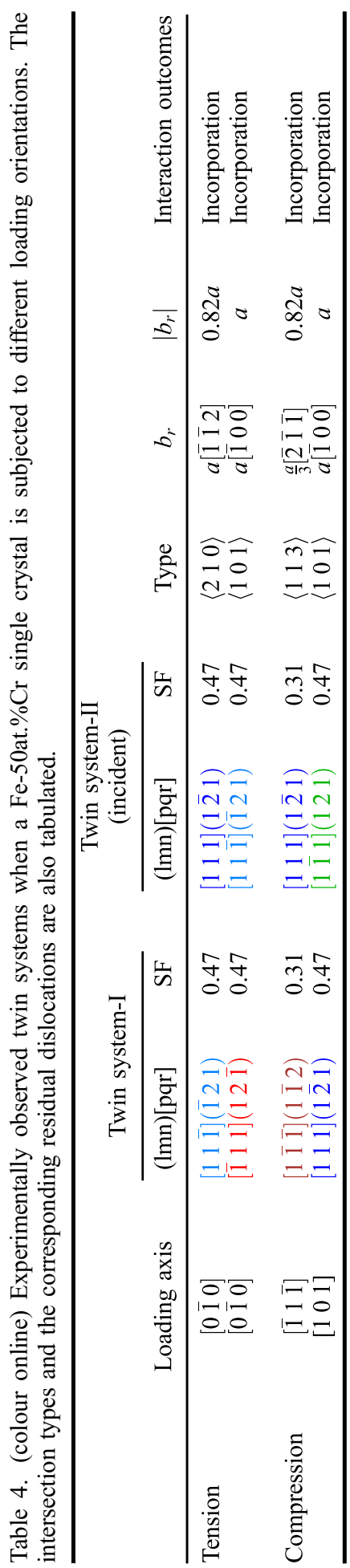


orientations largely helps to generate the interacting twins of certain intersection types experimentally. We choose the crystal orientations in such a way that the twins corresponding to the highest SF would nucleate and interact to yield the intersection type of our interest. In the next section, we will discuss these intersection types and their possible outcomes.

\section{Case (i) $\langle 110\rangle$ intersection type}

Figure 3 shows the stress-strain response of Fe-50at.\%Cr loaded along the $\left[\begin{array}{lll}1 & 0 & 1\end{array}\right]$ orientation in compression. The $x$-axis represents the average strain fields obtained utilizing DIC. A series of in situ DIC strain measurements (images marked A and B) show the onset of slip and twinning followed by twin migration at different stages of the loading curve. The strains displayed in the inset image marked $\mathrm{B}$ and point $\mathrm{C}$ represent the strain accumulation in the same regions that were linked to twin nucleation. Beyond point $\mathrm{C}$, another load drop is observed which indicates the activation of additional twins. DIC strain measurements show the strain localization on the $\left[\begin{array}{lll}1 & 1 & 1\end{array}\right]\left(\begin{array}{lll}1 & 2 & 1\end{array}\right)$ system corresponding to the highest SF. Slip nucleation at a CRSS $\left(\tau^{S}\right)$ of $87 \mathrm{MPa}$ is followed by the nucleation of $\left[\begin{array}{lll}1 & 1 & 1\end{array}\right]\left(\begin{array}{lll}1 & \overline{2} & 1)\end{array}\right)$ and $\left[\begin{array}{lll}1 & \overline{1} & 1\end{array}\right]\left(\begin{array}{lll}1 & 2 & 1\end{array}\right)$ twins (image B) at a CRSS $\left(\tau^{T}\right)$ of $202 \mathrm{MPa}$. Twinning is predominant in this loading orientation, and the increased hardening is manifested through twin-twin interactions and subsequent migration. An analysis on the interaction shows the formation of an $a\left[\begin{array}{lll}1 & 0 & 0\end{array}\right]$ residual dislocation which is indicative of the blockage of the incoming twin, and characterized by a preferential strain accumulation on one side of the existing twin $\left(\Delta \varepsilon_{T-T} \approx 1.5 \%\right.$ in Figure 4$)$. Each point in the strain measurement in Figure 4 corresponds to the average of the strain values obtained using ex situ DIC strain field. The overall mechanism can be described by the following reactions:

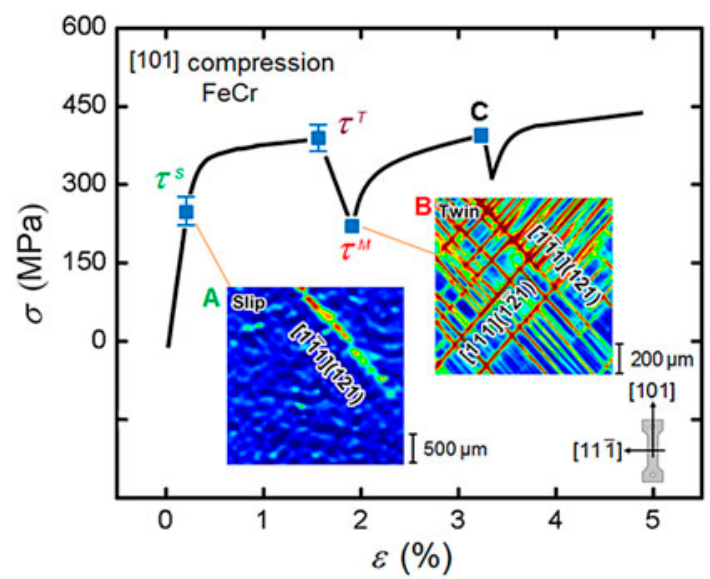

Figure 3. (colour online) Stress-strain response when a Fe-50at.\%Cr crystal is subjected to compression along the $\left[\begin{array}{lll}1 & 0 & 1\end{array}\right]$ orientation. Two twin systems corresponding to $\mathrm{SF}=0.47$ are observed to be activated (image B). $\tau^{S}$ represents the critical slip nucleation stress, $\tau^{T}$ represents the critical twinning stress and $\tau^{M}$ represents the critical twin migration stress (adapted from Ref. [12]). 


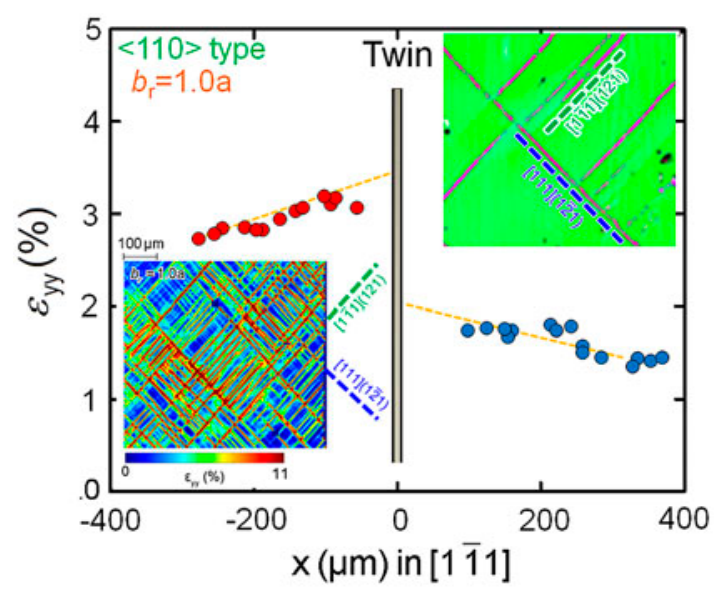

Figure 4. (colour online) EBSD (right figure) showing twin-twin interaction $\left(\begin{array}{llll}1 & 1 & 0\rangle\end{array}\right.$ intersection type) when the Fe-50at.\%Cr crystal is subjected to compression along the [ $\left.\begin{array}{lll}1 & 0 & 1\end{array}\right]$ orientation. The plot is accompanied by a high-resolution DIC contour plot (left figure) for the same loading orientation. The data (solid red and blue circles) show the post-interaction strain distribution on either side of the existing twin with respect to the position from the twin obtained using ex situ DIC. $\varepsilon_{m}$ represents the average strain field in the matrix.

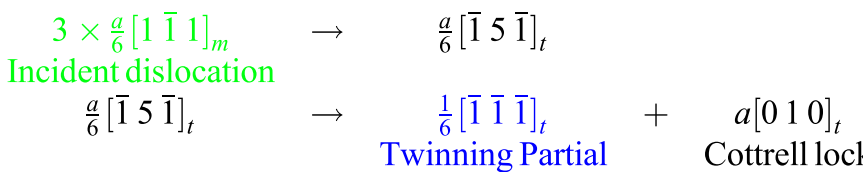

where subscripts $t$ and $m$ denote twin and matrix, respectively. The region of the twintwin interaction is paramount because it is associated with the residual dislocation developing high localized strain. This region largely influences the deformation process via hardening and in some cases, the sites for cleavage fractures [7,8,13-15]. Similar observations were made by Levasseur [7] and Mahajan [8] in single crystals of $\alpha$-iron where the authors characterize the $\left\langle\begin{array}{llll}1 & 1 & 0\end{array}\right\rangle$ intersection type to yield a residual dislocation of $1.0 \mathrm{a}$. The stress developed due to the localized strains is significant, and a higher stress would be required to migrate the twin.

The TEM images have been further utilized to study the $\left\langle\begin{array}{lllll}1 & 1 & 0\end{array}\right)$ type intersection. The micrograph illustrated in Figure 5 clearly shows the blockage of the incoming slips by an existing $\left[\begin{array}{lll}1 & 1 & 1\end{array}\right]\left(\begin{array}{lll}1 & 2 & 1\end{array}\right)$ twin producing similar results to that of twin-twin interaction of $\left\langle\begin{array}{llll}1 & 1 & 0\end{array}\right\rangle$ type. Slip-twin reactions can be regarded equivalent to the slip-twin interactions [5], and the expression (1) holds true for this case as well.

\section{Case (iii) $\langle 210\rangle$ intersection type}

Figure 6 shows the activation of the $\left[\begin{array}{llll}1 & 1 & \overline{1}\end{array}\right]\left(\begin{array}{lll}\overline{1} & 2 & 1\end{array}\right)$ and $\left[\begin{array}{lll}1 & 1 & 1\end{array}\right]\left(\begin{array}{lll}1 & \overline{2} & 1\end{array}\right)$ twins $(\mathrm{SF}=0.30)$ when the crystal is subjected to tension along the $[0 \overline{1} 0]$ orientation. Luca and 


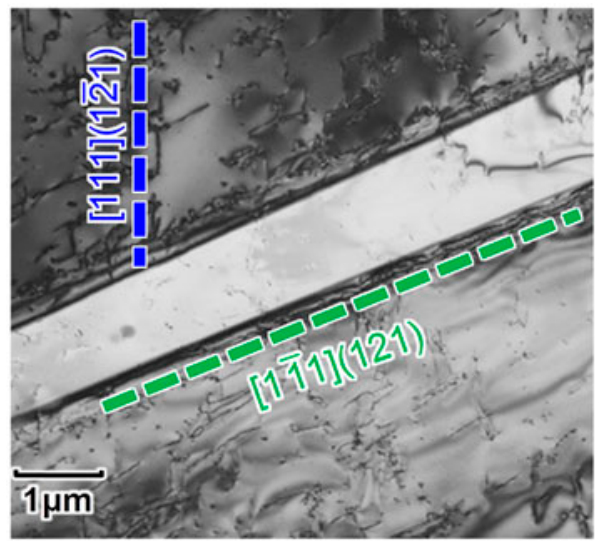

Figure 5. (colour online) TEM image of Fe-50at.\%Cr under $\left[\begin{array}{lll}1 & 0 & 1\end{array}\right]$ compression showing twinslip interaction. The incoming slips $\left[\begin{array}{lll}1 & 1 & 1\end{array}\right]\left(\begin{array}{lll}1 & \overline{2} & 1\end{array}\right)$ are completely blocked by the twin.

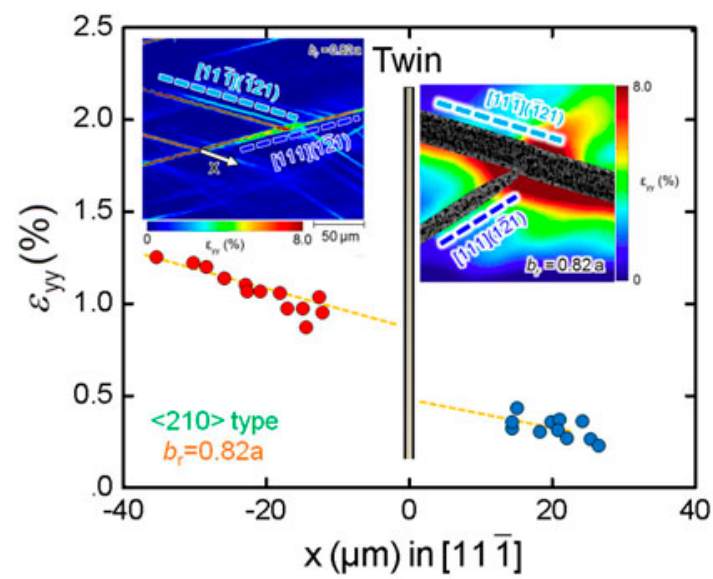

Figure 6. (colour online) Twin-twin interaction ( $\langle 210\rangle$ intersection type) when the Fe-50at.\%Cr crystal is subjected to tension along the $\left[\begin{array}{lll}0 & 1 & 0\end{array}\right]$ orientation. The data (filled circles) show the postinteraction strain distribution on either side of the existing twin with respect to the position from the twin. An incoming twin [ $\left.\begin{array}{lll}1 & 1 & 1\end{array}\right]\left(\begin{array}{lll}1 & \overline{2} & 1\end{array}\right)$ is blocked by an existing $\left[\begin{array}{lll}1 & 1 & \overline{1}\end{array}\right]\left(\begin{array}{lll}1 & 2 & 1\end{array}\right)$ twin resulting in a highstrain jump across the twin boundary.

co-workers [12] provide further details of the stress-strain response for this loading orientation. The interacting twins yield a dislocation of $0.82 a$, thus developing a high localized strain at the boundary. As displayed in Figure 6, since the incident twin is

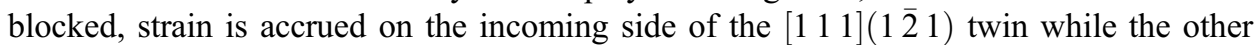
side has a comparatively lower strain (equal to the strain in the matrix). This indicates that no transmission has occurred across the twin boundary. A distinct drop in the strain observed on the other side of the twin generates a high strain jump $\left(\Delta \varepsilon_{T-T} \approx 0.5 \%\right)$ across the twin, and is attributed to the presence of the residual dislocation. The following reaction describes the overall process: 


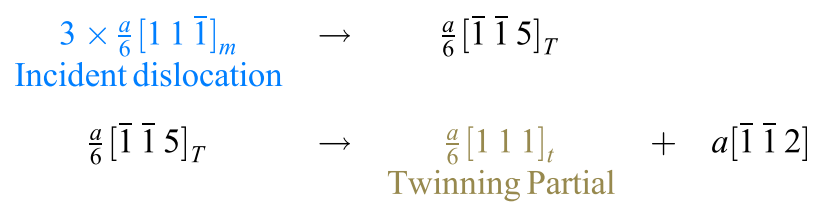

\section{Case (ii) $\left\langle\begin{array}{lll}3 & 1 & 1\rangle \text { intersection type }\end{array}\right.$}

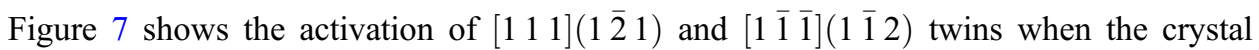
is subjected to compression along the $\left[\begin{array}{lll}\overline{1} & 1 & \overline{1}\end{array}\right]$ orientation. We utilize the high-resolution ex situ DIC strain measurements to study the residual strain field in the region of interacting twins. Figure 7 clearly shows that the incoming twin is blocked resulting in a high strain build-up predominantly in the interaction zone. Further analysis (Equation (3)) reveals that a residual dislocation of $0.82 a$ is left at the twin boundary. A high

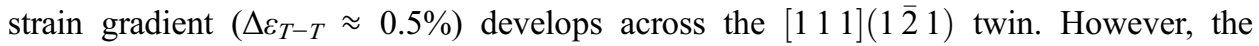
strain jump across the twin in this case $\left(\Delta \varepsilon_{T-T} \approx 0.5 \%\right)$ is comparatively lower than that for the $\left\langle\begin{array}{lll}1 & 1 & 0\end{array}\right\rangle$ case where $\Delta \varepsilon_{T-T} \approx 1.5 \%$. This is because the strain accumulation on the twin boundary is due to the residual dislocation, and the low magnitude of residual dislocation is observed in this case compared to the $\langle 1110\rangle$ intersection type. Thus, for an isolated twin-twin interaction, we would expect the twin migration stress to be lower for the $\left\langle\begin{array}{llll}1 & 1 & 3\end{array}\right\rangle$ intersection type compared to the $\left\langle\begin{array}{llll}1 & 1 & 0\end{array}\right)$ case. The overall mechanism can be summarized as follows:

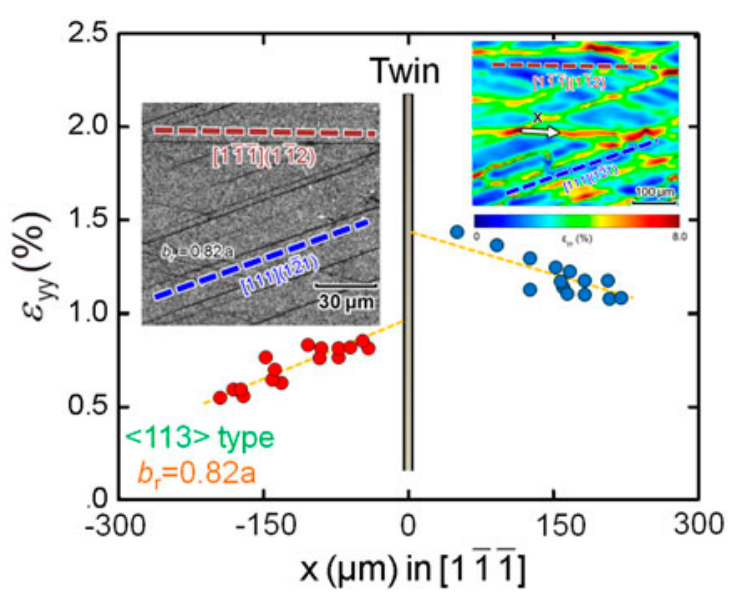

Figure 7. (colour online) Twin-twin interaction ( $\left.\begin{array}{llll}1 & 1 & 3\end{array}\right\rangle$ intersection type) when the $\mathrm{Fe}-50 \mathrm{at} . \% \mathrm{Cr}$ crystal is subjected to compression along the $\left[\begin{array}{lll}\overline{1} & 1 & \overline{1}\end{array}\right]$ orientation. The data show the post-interaction strain distribution on either side of the existing twin with respect to the position from the twin. An incoming twin $\left[\begin{array}{lll}1 & 1 & 1\end{array}\right]\left(\begin{array}{lll}1 & \overline{2} & 1\end{array}\right)$ is blocked by an existing [ $\left.\begin{array}{lll}1 & \overline{1} & \overline{1}\end{array}\right]\left(\begin{array}{lll}1 & \overline{1} & 2\end{array}\right)$ twin $\left(\left[\begin{array}{lll}1 & 1 & 3\end{array}\right]\right.$ type intersection) resulting in a high strain gradient across the twin boundary. 


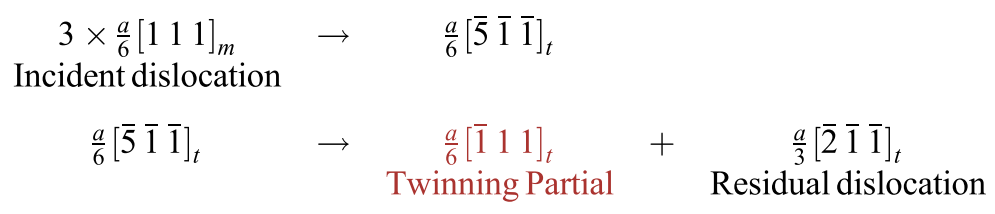

Several authors have reported that slip transmission is easily accomplished during twinslip or twin-twin interaction by means of dislocation dissociation at the twin-twin or twin-slip interface $[4,6-8,11]$. The stress concentration that builds up during twin-twin interaction is accommodated by slip occurring ahead of the twin. However, the slip bands may not be always present after the interaction. When an incident twin is terminated on an existing twin in the crystal, a high stress concentration develops primarily due to the residual dislocation. Further dissociation either requires a large magnitude of resolved shear stress on the slip system within the twin or the dissociation should be energetically favourable. In bcc crystals, experiments showing pure transmission at the expense of the residual dislocation across the twin have been reported. Particularly in the $\langle 210\rangle$ intersection type $\left(b_{r}=0.82 a\right)$, an increase in the external stress may possibly facilitate the dissociation of the residual dislocation into slip that may transmit across the twin boundary. However, in the present experiments, we do not observe such a case.

\section{MD simulation}

To gain further insight into the results, MD simulations were undertaken for the bcc case utilizing Fe potential [18]. A periodic box was created using LAMMPS where a 10-layer thick twin was introduced. Twin nucleation occurred from a prescribed source under monotonic loading of single crystals with the same orientation as the specimens. The source is placed at a sufficiently large distance from the twin so that the stresses of the twin and the source do not interact. Before deforming the crystal, the total energy of the system is minimized. The energy evolution of the system is traced so as to ensure that the system is at a minimum energy before deforming. During the dynamics process, the crystal is subjected to a strain of $20 \%\left(\dot{\epsilon}=10^{8} \mathrm{~s}^{-1}\right)$ at a temperature of 10 $\mathrm{K}$. A series of visualization tools were utilized to examine the three loading orientations, the reactions and the resulting mechanical response. The results are consistent with the experiments. Specifically, from the present calculations employing MD simulations, higher migration stresses were observed for the $1.0 a$ cases compared to the $0.82 a$ case (Table 5). In the simulation, two cases corresponding to $\left\langle\begin{array}{llll}1 & 1 & 0\end{array}\right\rangle$ and $\left\langle\begin{array}{llll}1 & 1 & 3\end{array}\right\rangle$ intersection types were considered. This allows the observation of twin migration process for the isolated cases of different residual burgers vectors and provides a better understanding of the mechanisms. We also checked the response with the use of a Fe-50at.\% $\mathrm{Cr}$ potential [19] and the trends are similar. The results are summarized in Figures 8 and 9 , and show the blockage of the incoming twin followed by the incorporation of the twinning dislocations.

Table 5 shows the simulation results for $\left\langle\begin{array}{llllll}1 & 1 & 0\end{array}\right)$ and $\left\langle\begin{array}{llll}1 & 1 & 3\end{array}\right\rangle$ intersecting twins and the corresponding residual dislocations. As shown in Figure 8, when the crystal is subjected to tension along the $\left[\begin{array}{lll}0 & \overline{1} & 0\end{array}\right]$ orientation, $\left(\begin{array}{lll}1 & 2 & \overline{1}\end{array}\right)\left[\begin{array}{lll}\overline{1} & 1 & 1\end{array}\right]$ and $\left(\begin{array}{lll}\overline{1} & 2 & 1\end{array}\right)\left[\begin{array}{lll}1 & 1 & \overline{1}\end{array}\right]$ twins are activated, the interaction of which produces a residual dislocation of $1.0 a$ and a 


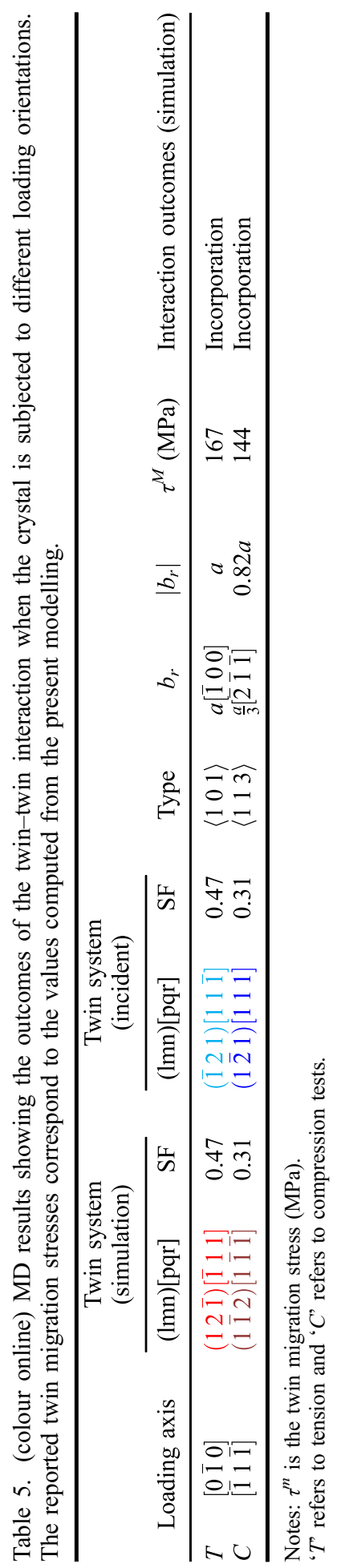




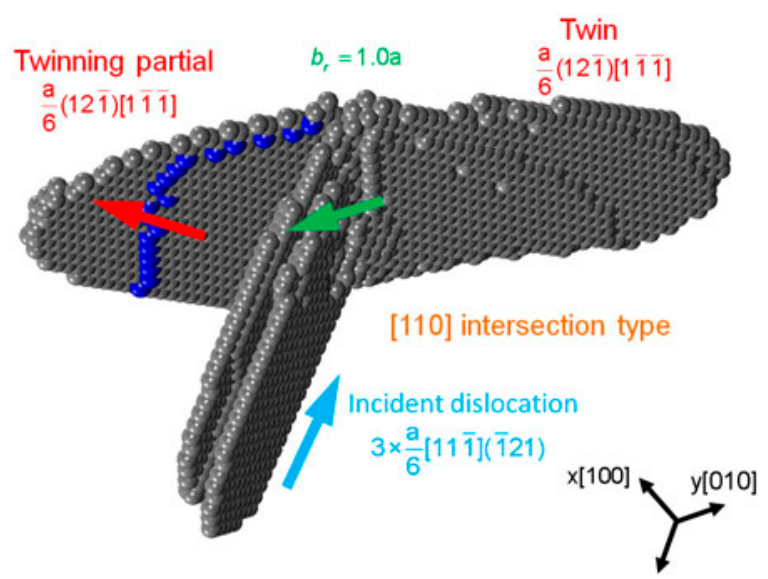

Figure 8. (colour online) MD simulation showing twin-twin interaction ([ $\left[\begin{array}{lll}1 & 1 & 0\end{array}\right]$ intersection type) when the crystal is subjected to tension along the $[0 \overline{1} 0]$ intersection. The incident twin $\left(\begin{array}{lll}\overline{1} & 2 & 1\end{array}\right)\left[\begin{array}{lll}1 & 1 & \overline{1}\end{array}\right]$ is completely blocked and a twinning dislocation glides along the twin boundary. The residual dislocation formed at the interface is $1.0 a$.

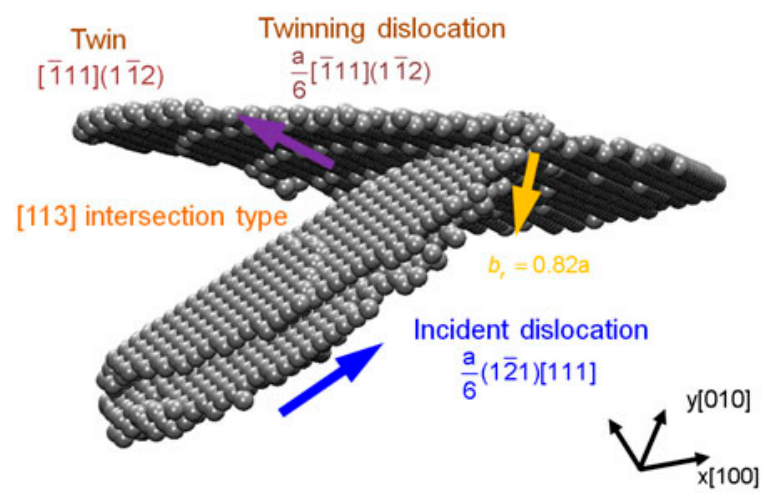

Figure 9. (colour online) MD simulation showing twin-twin interaction ([1 113$]$ intersection type) when the crystal is subjected to compression along the [1 111$]$ orientation. The incident twin $\left(\begin{array}{lll}1 & \overline{2} & 1\end{array}\right)\left[\begin{array}{lll}1 & 1 & 1\end{array}\right]$ is completely blocked by $\left(\begin{array}{lll}1 & \overline{1} & 2\end{array}\right)\left[\begin{array}{lll}1 & 1 & 1\end{array}\right]$, and a twinning partial is formed that glides along the twin boundary. The residual dislocation formed at the interface is $0.82 a$.

twinning partial that glides along the twin boundary thus translating the boundary by one layer. Consistent with the experimental observations, the twin is completely blocked and the process can be summarized with expression (1) described earlier. Similarly,

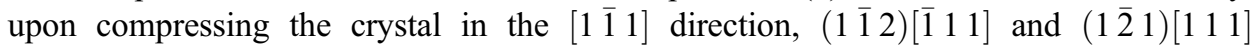
twins are activated corresponding to the highest SF (0.31) in this loading orientation (Figure 9). The $\left\langle\begin{array}{llll}1 & 1 & 3\end{array}\right\rangle$ type twin intersection results in the blockage of the incident twin due to the formation of the residual dislocation of large magnitude $(0.82 a)$. The overall mechanism for this case can be represented by expression (3). 


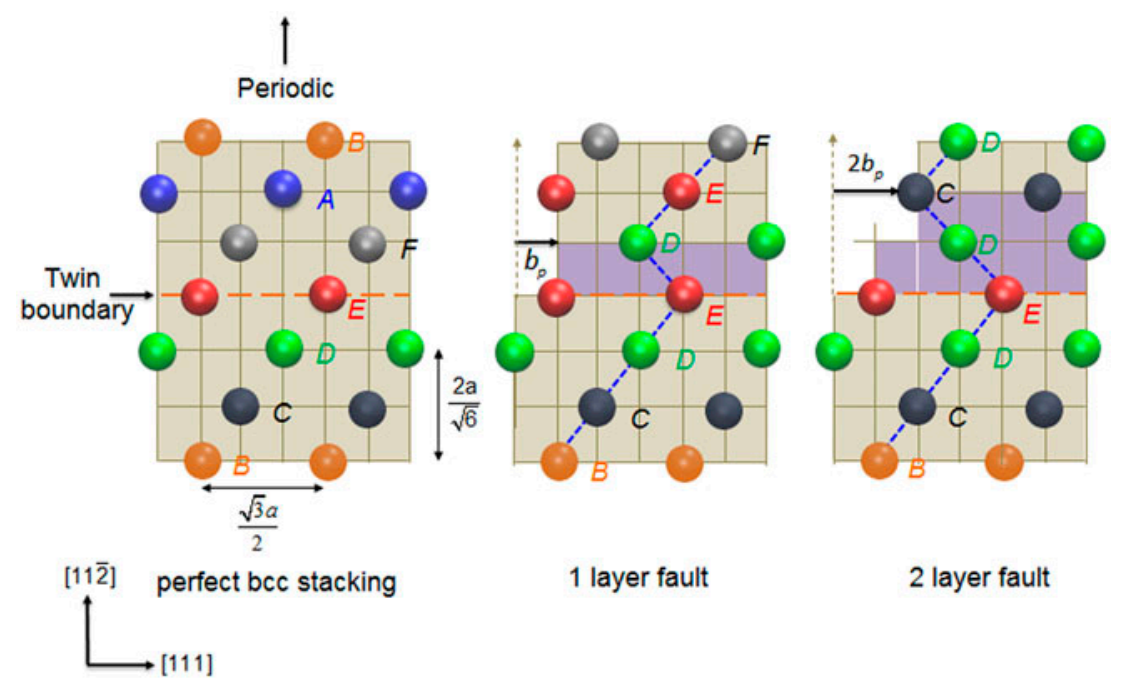

Figure 10. (colour online) The procedure for establishing the GPFE curve in bcc crystal. The $\left\{\begin{array}{llll}1 & 1 & 2\end{array}\right\}\left\langle\begin{array}{llll}1 & 1 & 1\end{array}\right\rangle$ stacking sequence is shown. Atoms on different $\left\{\begin{array}{llll}1 & 1 & 2\end{array}\right\}$ planes are represented by different colours. The arrows indicate the passage of the dislocation $b_{p}=\frac{a}{6}\langle 111\rangle$ on successive $\left\{\begin{array}{lll}1 & 1 & 2\end{array}\right\}$ planes creating one-layer and two-layer stacking faults.

We are well aware through present experimental observations and simulation that the residual dislocations are associated with high localized strains and stresses that significantly increase the stress required for twin migration. It is now important to quantify these effects on the twin migration. We will first investigate the effects of $b_{r}$ in modifying the twinning energy pathways (GPFE) in bcc crystals, and then develop an expression utilizing the modified energy pathways to quantify the twin migration stress as a function of $b_{r}$ in the following sections. In bcc crystals, the GPFE is the energy per unit area required to shear one elastic half of the crystal with respect to the other half by a displacement of $\frac{a}{6}\left\langle\begin{array}{llll}1 & 1 & 1\end{array}\right\rangle$ on successive $\left\{\begin{array}{lll}1 & 1 & 2\end{array}\right\}$ planes. We will use the term 'modified GPFE' in this paper to refer to the GPFE calculated in the presence of the residual dislocation unlike the GPFE usually obtained for a perfect crystal (Figure 10).

\section{Twinning energy pathways (GPFE)}

The multilayer stacking fault (twin) in bcc crystals is a result of the passage of the $\frac{a}{6}\left\langle\begin{array}{llll}1 & 1 & 1\end{array}\right\rangle$ dislocations on successive $\left\{\begin{array}{lll}1 & 1 & 2\end{array}\right\}$ planes [16]. In most bcc metals, a three-layer stacking fault is considered to be a twin nucleus [17], while additional faults result in the growth of the twin. To obtain the GPFE landscape for Fe, a simulation box consist-

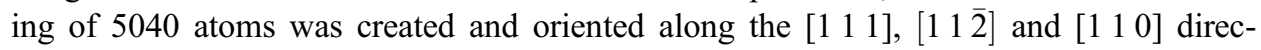
tions. The box was relaxed at a temperature of $10 \mathrm{~K}$. Periodicity was invoked across the box in [ $\left[\begin{array}{lll}1 & 1 & 1\end{array}\right]$ and $\left[\begin{array}{lll}1 & 1 & 0\end{array}\right]$ directions while the $\left[\begin{array}{lll}1 & 1 & \overline{2}\end{array}\right]$ direction was delimited by a free surface. During the process of shearing consecutive $\left\{\begin{array}{llll}1 & 1 & 2\end{array}\right\}$ planes by a displacement of

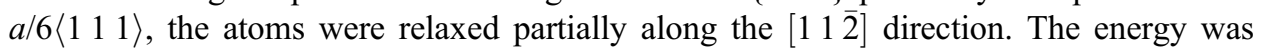




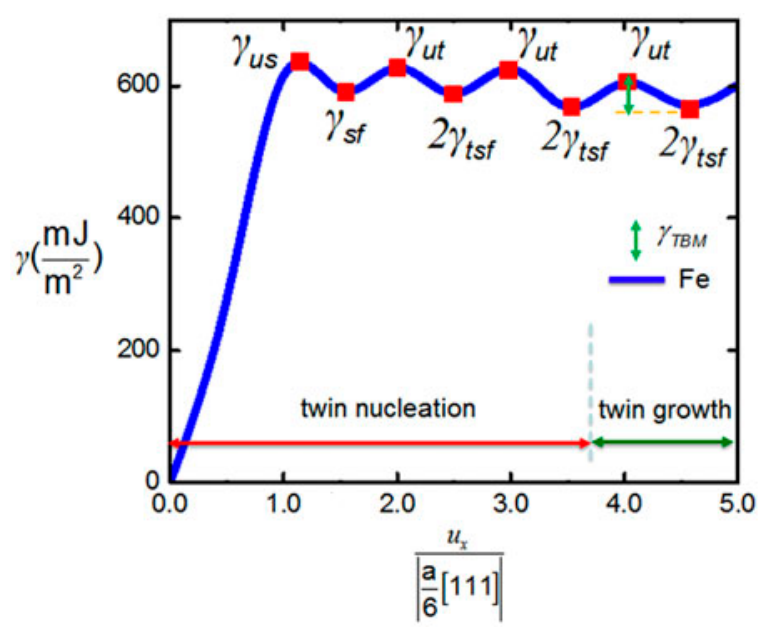

Figure 11. (colour online) GPFE for a bcc $\left\{\begin{array}{llllll}1 & 1 & 2\end{array}\right\}\left\langle\begin{array}{llll}1 & 1 & 1\end{array}\right\rangle$ system. The regions of twin nucleation and twin migration are also shown. The $x$-axis is given in units of the Burgers vector.

traced for each sheared $\left\{\begin{array}{llll}1 & 1 & 2\end{array}\right\}$ plane corresponding to an $\frac{a}{6}\left\langle\begin{array}{llll}1 & 1 & 1\rangle\end{array}\right)$ displacement, and the stacking fault energies corresponding to normalized displacements $\left(\frac{u_{X}}{b_{p}}\right)$ were calculated using the following equation:

$$
E_{\mathrm{SF}}=\frac{E_{\text {sheared }}-E_{\text {perfect }}}{A}
$$

where $E_{\text {sheared }}$ is the energy of the crystal corresponding to an $\frac{a}{6}\left\langle\begin{array}{lll}1 & 1 & 1\end{array}\right\rangle$ displacement for each sheared $\left\{\begin{array}{lll}1 & 1 & 2\end{array}\right\}$ plane, $E_{\text {perfect }}$ is the energy of the perfect crystal and $A$ is the total area of the $\left\{\begin{array}{lll}1 & 1 & 2\end{array}\right\}$ plane of the simulation box. The GPFE curve for Fe was obtained using an EAM potential [18] while the curves for other bcc alloys were obtained in a similar manner employing various other potentials $[19,20]$. The potentials used in the present calculations yield physical properties (such as elastic constants, lattice parameters, cohesive energy, solid solution lattice parameters, etc.) in a reasonable agreement with the experimental data or other empirical methods.

Figure 11 shows the GPFE for Fe where $\gamma_{U S}$ refers to the unstable stacking fault energy and represents the energy barrier that a dislocation should overcome to create the first layer stacking fault. Similarly $\gamma_{\mathrm{sf}}$ is the stable stacking fault energy, $\gamma_{\mathrm{ut}}$ is the unstable twinning fault energy, $\gamma_{\mathrm{TBM}}$ is the twin migration energy and $2 \gamma_{\text {tsf }}$ is the stable twin stacking fault energy. The twin boundary migration energy in Figure 11 can be decomposed as follows:

$$
\gamma_{\mathrm{UT}}=2 \gamma_{\mathrm{TSF}}+\gamma_{\mathrm{TBM}}
$$

As shown in Figure 11, the energy profile becomes stable beyond the third layer which implies that once a third layer twin is nucleated in bcc Fe, there is no change in the stacking fault energies, $\gamma_{\mathrm{ut}}$ and $2 \gamma_{\mathrm{TSF}}$ when a dislocation glides over an existing fault to create an additional layer (twin migration). 


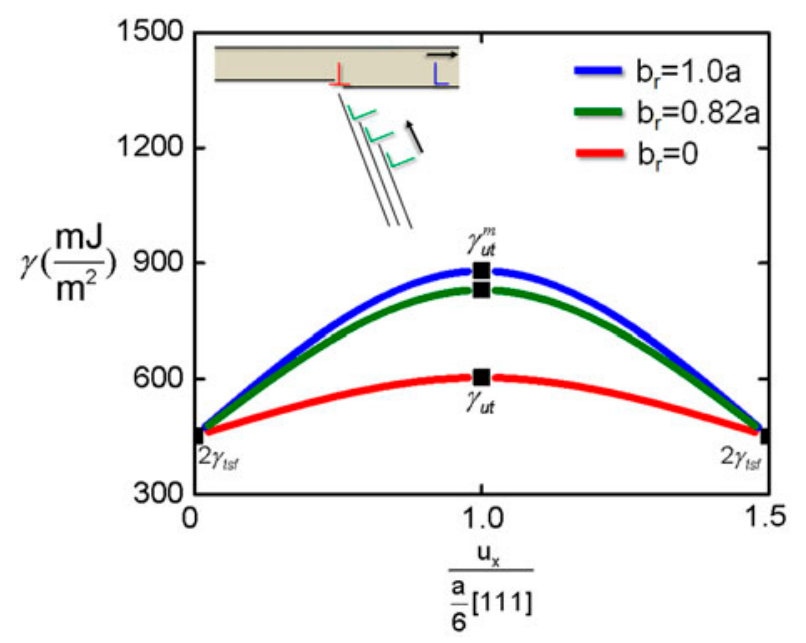

Figure 12. (colour online) The effect of residual dislocation $\left(b_{r}\right)$ on the GPFE for a bcc Fe crystal. It is observed that $\gamma_{\text {ut }}$ increases with an increase in the magnitude of the residual dislocation. The energy values correspond to a 10-layer twin.

The role of energy barrier in twin nucleation in bcc metals and alloys has been discussed extensively and utilized to predict the twinning stress [9]. To add an extra layer to the existing twin boundary, the dislocation has to overcome the energy barrier $\gamma_{\mathrm{ut}}$. As said earlier, the residual dislocation left at the twin boundary increases the energy barrier $\gamma_{\mathrm{ut}}$ [10], thus making the glide of the dislocations more difficult along the twin boundary. We will investigate the changes in the energy barrier in the presence of the residual dislocation in this work. For this, we compute the modified GPFE in the presence of the residual dislocation, the details of which are presented in Appendix A1. Figure 11 shows the GPFE and Figure 12 shows the modified GPFE for bcc Fe obtained through MD where $\gamma_{\mathrm{ut}}^{m}$ is seen to increase with increase in the magnitude of the residual dislocation $\left(\gamma_{\mathrm{ut}}^{m}>\gamma_{\mathrm{ut}}\right)$. The increase in $\gamma_{\mathrm{ut}}^{m}$ reflects that a higher stress (twin migration stress) is required to glide the dislocation along the twin boundary in the presence of the residual dislocation when compared to the case when no residual dislocation is left at the twin boundary. For further analysis, we express the modified GPFE depicted in Figure 12 using the sine analytical functions as follows:

$$
\begin{gathered}
\left.\gamma_{\text {modifiedGPFE }}(\lambda(x))=\frac{1}{2}\left(\gamma_{\mathrm{ut}}^{m}+\gamma_{\mathrm{tsf}}\right)+\left(\gamma_{\mathrm{UT}}^{m}-\frac{\gamma_{\mathrm{ut}}^{m}+\gamma_{\mathrm{tsf}}}{2}\right) \sin [2 \pi(\lambda(x)-1.21)]\right) \\
\text { for } 10 \leq \lambda(x)<11
\end{gathered}
$$

In the above equations, $\lambda$ is the normalized shear displacement of the two halves of the crystal due to the passage of the dislocation $b_{p}=a / 6\left\langle\begin{array}{llll}1 & 1 & 1\rangle\end{array}\right)$ and $\gamma_{\mathrm{UT}}^{m}$ is the modified unstable twinning fault energy. In the following section, we use the modified energy pathways (modified GPFE) and incorporate it with the elastic interactions of the twinning dislocations to develop an expression that quantifies the effect of $b_{r}$ on the twin migration stress. 


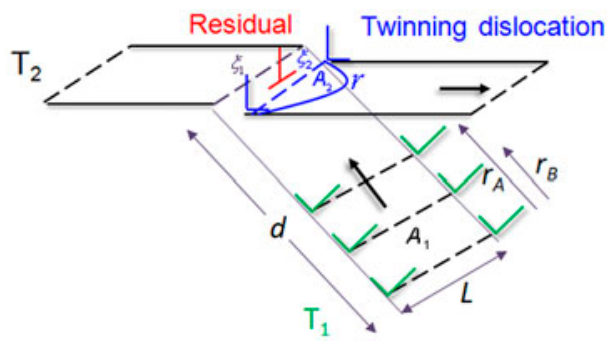

Figure 13. (colour online) Schematic showing the twin-twin interaction in a bcc crystal. A residual dislocation is left at the twin boundary as a result of the interaction. A twinning partial generated at the twin boundary translates the boundary by one layer.

\section{Mesoscale modelling of twin migration stress}

The resistance of the grain boundaries (including $\sum 3$ boundaries) in dislocation slip transmission has been studied earlier by Koning and co-workers [21]. Through the line tension model, the authors were able to develop a set of geometrical parameters (such as SF, standoff ratio and magnitude of residual dislocation) that govern slip transmission across different types of grain boundaries. However, in this study, we characterize the resistance of twin to dislocation glide along its boundary in terms of the twin migration stress, i.e. a higher twin migration stress reflects a higher resistance to dislocation glide along the twin boundary. We do so by considering the total energy of the interacting dislocations and minimizing the energy to obtain the critical stress required for twin migration. The details of the calculations will be discussed next.

A schematic of twin-twin interaction is depicted in Figure 13. Consider that a twin $T_{1}$ of width $L$ nucleates from the source at a CRSS of $\tau^{T}$. The twin nucleus $T_{1}$ of area $A_{1}$ consists of three partial dislocations that are situated at positions $r_{A}$ and $r_{B}$ from the source $\left(r_{C}=0\right)$. The twin interacts with an existing twin $T_{2}$ situated at distance $d$ from the source. Assume that the incoming twin $\left(T_{1}\right)$ touches the twin $\left(T_{2}\right)$ at two points in the line of intersection separated by distance $\zeta_{1}$. A residual dislocation $b_{r}$ is left at the intersection line $\zeta_{2}$ as a result of the interaction. Under sufficient applied stress, the partial dislocation with Burgers vector $b_{2}$ is assumed to generate through the line of intersection $\zeta_{2}$ of the interacting twins. Consider that the activated dislocation in twin $T_{2}$ is pinned at two points separated by $\zeta_{2}$. The pinned dislocation segment acts as a FrankRead source, and generates dislocations that cause the twin to migrate. Under the sufficient stress, the dislocation segment $b_{2}$ bows with a finite radius $r$. The radius $r$ of the semicircular dislocation segment that sweeps an area $A_{2}$ on the verge of migration can be calculated as follows:

$$
r=\frac{\zeta_{2}}{2}=\frac{\kappa}{\tau_{2} b_{2}}
$$

where $\kappa=\frac{G b_{2}^{2}}{4 \pi} \ln \left(\frac{R}{w}\right), b_{2}$ is the Burgers vector of the migrating partial dislocation and $G$ is the shear modulus of the $\left\{\begin{array}{llll}1 & 1 & 2\end{array}\right\}\left\langle\begin{array}{llll}1 & 1 & 1\end{array}\right\rangle$ twinning system. It is well known that the critical stress required for the activation of the Frank-Reed source is given by: 


$$
\tau_{\text {critical }}=\frac{2 \kappa}{b \zeta_{2}}
$$

The critical stress in Equation (5) is in fact, the stress required for migrating the twin by one layer. It is seen from Equation (5) that the twin migration stress is dependent on the burgers vector of the dislocation and the separation distance $\zeta_{2}$ at which the dislocation segment is pinned (see Appendix A2 for detail calculations of $\zeta_{2}$ ). In all the cases we consider, $b_{2}$ is the twinning partial which is equal to $\frac{a}{6}\langle 111\rangle$. We choose $L$ to be consistent throughout the calculations by assuming the incident twin with a constant width. We predict the critical stress required for twin migration based on the geometrical parameters of the twin (width and length) and the magnitude of the residual dislocations obtained through MD. The total energy of the configuration in Figure 13 is the sum of (i) the interaction energy of the incident twinning dislocations; (ii) the line energies of the incident and the outgoing dislocations; (iii) the energy landscape associated with the incoming dislocations which is quantitatively represented by the GPFE; (iv) the modified energy landscape associated with the outgoing dislocation; (v) the energy of the residual dislocation; and (vi) minus the work done by the resolved stresses to move the incident and outgoing dislocations. The total energy expression can be written as follows:

$$
\begin{aligned}
U_{\text {total }}= & E_{\text {interaction/incident }}+E_{\text {line } / \text { incident }}+E_{\text {line } / \text { outgoing }}+E_{\text {residual }}+E_{\text {incidentGPFE }} \\
& +E_{\text {modifiedGPFE }}-W_{\text {incident }}-W_{\text {outgoing }} \\
= & -\frac{G b_{1}^{2}}{2 \pi}\left\{\ln \left[\frac{r_{B}-r_{A}}{r_{o}}\right]+\ln \left[\frac{r_{B}}{2 r_{o}}\right]+\ln \left[\frac{r_{A}}{r_{o}}\right]\right\} \zeta_{1}+\frac{G b_{1}^{2}}{4 \pi} \ln \left(\frac{R}{w}\right)\left\{2 d+\zeta_{1}\right\} \\
& +\frac{G b_{2}^{2}}{4 \pi} \ln \left(\frac{R}{w}\right)\left\{l_{2}-\zeta_{2}\right\}+\frac{G b_{r}^{2}}{4 \pi} \ln \left(\frac{R}{w}\right) \zeta_{2}+A_{1} \int_{0}^{N_{1}} \gamma_{\text {incidentGPFE }} d \lambda \\
& +A_{2} \int_{N_{2}}^{N_{2}+1} \gamma_{\text {modifiedGPFE }} d \lambda-\tau_{1} b_{1} A_{1}-\tau_{2} b_{2} A_{2}
\end{aligned}
$$

where $b_{1}$ and $b_{2}$ are the Burgers vector of the incoming and the outgoing dislocations, respectively, and $N_{1}$ and $N_{2}$ are their corresponding number of layers. The term $\gamma_{\text {incidentGPFE }}$ represents the GPFE of the three layer twin nucleus $T_{1}$ and $\gamma_{\text {modifiedGPFE }}$ represents the modified GPFE for twin $T_{2}$ obtained in the presence of the residual dislocation (see Figure 12). The parameters $r_{A}$ and $r_{B}$ represent the initial positions of the dislocations of the twin $T_{1}$ from the source $\left(r_{C}=0\right)$, and are calculated from the stacking fault energy and the shear modulus $(G)$ of the $\left\{\begin{array}{llllllllllllll}1 & 1 & 1 & 1\end{array}\right\}$ system (see Appendix A2 for the calculation of $r_{A}$ and $r_{B}$ ). The term $r_{o}$ represents the equilibrium distance between the partial dislocations $A$ and $B$ under zero external stress, and is equal to $r_{A} / 2$ [22] and $l_{2}$ represents the semicircular arc length of the activated partial dislocation $b_{2}$. Similarly, the term $R$ is a measure of the dimension of the crystal containing the dislocation and is approximated as $500 \mathrm{w}$ both for the incident and outgoing dislocations, where $w$ is the dislocation core width [23,24]. This choice $\left(\frac{R}{w}=500\right)$ results in the well-known $\frac{1}{2} G b^{2}$ expression representing the elastic energy of the dislocation. Nevertheless, the ratio of the shear stress drop ( $\frac{\tau^{M}}{\tau^{N}}$ values) is not affected by the expected range of choice of $\frac{R}{w} . N_{1}$ is equal to 3 and $N_{2}$ is equal 10 in the present analysis as we observed a three-layer twin nucleating from the source that interacted with a 10-layer twin introduced in the simulation. Therefore, $\gamma_{\text {modifiedGPFE }}$ accounts for 
the energy barrier that a partial dislocation gliding on the $N_{2}$-th layer of the twin has to overcome in order to translate the twin to the $\left(N_{2}+1\right)$ th layer in the presence of the residual dislocation. For a given pair of incoming and outgoing dislocations with resolved shear stresses $\tau_{1}$ and $\tau_{2}$, a stable configuration can be determined by minimizing Equation (6) with respect to $\zeta_{1}$ and $\zeta_{2}$. In case the incoming dislocation incorporates in $T_{2}, \tau_{2}=\tau_{m}$ is defined to be the twin migration stress as it corresponds to the stress required to move the twin boundary by one layer. The critical stress for twin migration is then calculated as follows (refer to the Appendix A2 for detail calculations):

$$
\begin{aligned}
\frac{\tau^{M}}{\tau^{N}}= & -\frac{\frac{\pi}{2} \cdot \frac{G b_{2}^{2}}{4 \pi} \ln \left(\frac{R}{W}\right) \cdot \int_{N_{2}}^{N_{2}+1} \gamma_{\text {modifiedGPFE }} d \lambda}{2 b_{2} \cdot \frac{G b_{r}^{2}}{4 \pi} \ln \left(\frac{R}{W}\right)+(\pi-1) \frac{G b_{2}^{3}}{4 \pi} \ln \left(\frac{R}{W}\right)} \\
& \cdot\left\{\frac{b_{1} r_{A}}{-\frac{G b_{1}^{2}}{2 \pi}\left\{\ln \left[\frac{r_{B}-r_{A}}{r_{o}}\right]+\ln \left[\frac{r_{B}}{2 r_{o}}\right]+\ln \left[\frac{r_{A}}{r_{o}}\right]\right\}+\frac{G b_{1}^{2}}{2}+r_{A} \int_{0}^{N_{1}} \gamma_{\text {incidentGPFE }} d \lambda}\right\}
\end{aligned}
$$

Several authors $[9,10]$ have discussed the role of energy pathways associated with a growing twin (twin migration). Expression (7) elucidates the dependence of the twin migration stress on the magnitude of the residual dislocation and the energy barriers associated with the twin migration process. In order to predict the twin migration stress accurately, it is important to consider the modified GPFE. Since the higher magnitude of the residual dislocation elevates the energy barrier $\gamma_{\mathrm{UT}}^{m}$ (see Figure 12), the increase in energy should be reflected in the total energy expression (6).

\section{Discussion of the results}

Figure 14 illustrates the magnitude of the twin migration stress as a function of $b_{r}$ obtained utilizing Equation (7) for a number of bec metal and alloys. The results show

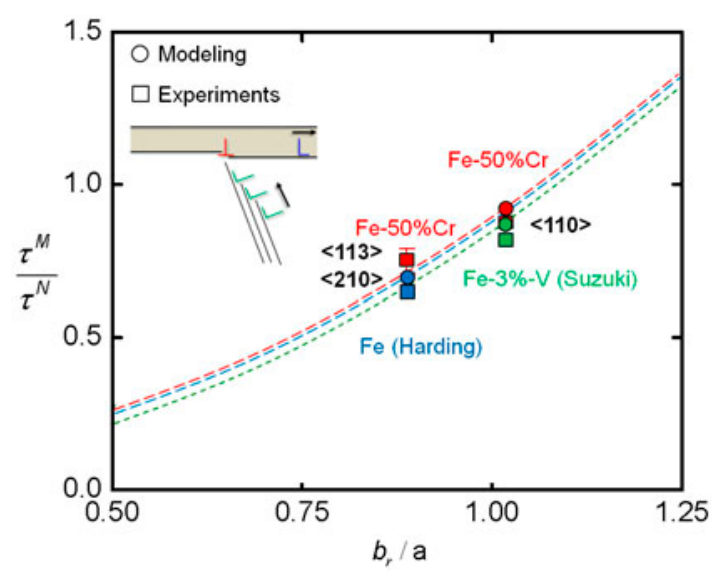

Figure 14. (colour online) Twin migration stress as a function of $b_{r}$ for an isolated twin-twin interaction. $\frac{\tau^{M}}{\tau^{N}}$ represents the ratio of twin migration stress to the twin nucleation stress. ' $a$ ' is the lattice constant of the bcc crystal. The coloured dashed lines correspond to $\mathrm{Fe}-50 \mathrm{at} . \% \mathrm{Cr}, \mathrm{Fe}$ $[25,26]$ and Fe-3at.\%V [27]. Note that the experimental $\tau^{M}$ and $\tau^{N}$ levels were shown in Figure 3 for $\mathrm{Fe}-50$ at. $\% \mathrm{Cr}$. 
that subsequent to twin-twin interactions, the migration stress for twinning, which is a measure of the resistance to twin motion, is proportional to the residual burgers vector. Specifically, from the calculations using MD simulations, higher migration stresses were observed for the $1.0 a$ case compared to the $0.82 a$ cases, as shown in Table 2. This allows the observation of twin migration for the isolated cases of different residual burgers vectors and provides a better understanding of the mechanism.

It is seen from Figure 14 that the stress required to migrate the twin increases significantly with increasing $b_{r}$, thus having a profound effect on the hardening behaviour. Similar results have been obtained through MD simulations [21] for grain boundaries where the resistance for slip transmission increases with an increase in the magnitude of the residual dislocation. The sessile residual dislocations in the above expression which are left at the twin boundary increase the local stress field due to the disarrangement of the atoms in the vicinity of the interacting zone. The DIC strain measurements shown in Figures 4-7 are a direct measure of the distortion created by these residual dislocations. Furthermore, the calculation of the modified GPFE using atomistic methods shows that the energy parameter, $\gamma_{\mathrm{TBM}}$, which represents the energy barrier that the partial dislocation has to overcome during twin growth (migration) is increased due to residual dislocation. Both the experimental and MD simulation methods are coupled in this work to show a strong correlation of the increase in the local stress field (and thus the twin migration stress) with an increase in the magnitude of the $b_{r}$. Thus, we forward that the increase in twin migration stress is manifested due to (i) the local stress field generated by the residual $b_{r}$, and (ii) the subsequent elevation in the modified energy barrier $\gamma_{\mathrm{TBM}}$.

The results (Figures 4-7) clearly show the strain gradients that develop in pristine single crystals as a result of twin-twin interactions. The gradients are a function of the residual burgers vector that form at the intersections and span across the twin boundaries. Consequently, they represent long-range strain fields that will influence the overall deformation behaviour as well as the local behaviour that could trigger failure. As shown in this study, based on the analysis of the dislocation reactions, the residual burgers vector has a magnitude near $1.0 a$ where ' $a$ ' is the lattice spacing. Previous studies that calculated the residual burgers vectors on polycrystals were performed on fcc crystals [28-32] using the $\left\{\begin{array}{lll}1 & 1 & 1\end{array}\right\}$ slip. The important distinction in the present study is the activation of $\left\{\begin{array}{lll}1 & 1 & 2\end{array}\right\}$ planes and the use of single crystals, and the present results have been obtained with high-resolution DIC. It would not be possible to obtain the local variations with the standard DIC methods. Special attention has been devoted to the preparation of the samples with surface patterning methods which allow the achievement of small subset sizes. It is particularly important to note that the twins span across the entire specimen, but their width is small (less than 100 microns) in most cases; therefore, high-resolution DIC provides accurate information on the gradients.

The strain distribution across the twin boundary observed in experiments has been rationalized in terms of the residual dislocations left at the boundary as a result of the interaction. The systems of the interacting twins are first identified in experiments and simulations, and the residual dislocations are calculated. The DIC strain measurements show a high localized strain in $\left\langle\begin{array}{lll}1 & 1 & 0\rangle\end{array}\right.$ type intersection (Figure 4) compared to the $\left\langle\begin{array}{lll}1 & 13\end{array}\right\rangle$ and $\langle 210\rangle$ intersection types (Figures 6 and 7 ) due to the higher magnitude of residual dislocation in the former. The importance of $b_{r}$ is highlighted in the present work because it contributes to an increased hardening by distorting the local stress field, 
and increasing the energy barriers for twin migration. Nonetheless, the residual dislocations are associated with high strains in the vicinity of the twin boundaries and can be the preferred sites of cleavage fracture. The association of twin and crack initiation in bcc metals has been well documented in most metals [7,8,13-15,33]. Twin-twin interactions can be beneficial for the strengthening of the materials, and on the other side, the intersection regions can promote high strain and stress localizations [34-36], especially when a twin is blocked by another existing twin. Experimental studies show that in some cases, twins allow the transmission of the incident dislocations while in other cases, blockage is observed. While blocked dislocations are associated with higher strain and confirm a residual dislocation of higher magnitude, transmission is accomplished by the dissociation of the residual dislocation whereby the stress is relieved in the interaction zone. Therefore, in cases where transmission is observed, no sessile residual dislocations are present. In the present calculations, when $b_{r}$ approaches zero, $\frac{\tau^{M}}{\tau^{T}}$ becomes approximately 0.15 for $\mathrm{Fe}-50 \mathrm{at} . \% \mathrm{Cr}$, which reflects that the stress required for twin migration is much lower when no residual dislocation is left at the twin boundary. The critical resolved shear stress in this case $\left(b_{r}=0\right)$ has to overcome the lower $\gamma_{\mathrm{TBM}}$ for twin migration thus yielding a lower twin migration stress, which otherwise would be higher $\left(\gamma_{\mathrm{TBM}}^{m}>\gamma_{\mathrm{TBM}}\right)$ in the presence of the residual dislocation.

The interaction of dislocations with different grain boundaries (including the highsymmetry $\sum 3$ boundary) has been well studied using experimental and atomistic methods [4-6,8-10,21]. The geometrical criteria forwarded by Shen [37] and Lee [38] explain the degree of easiness for a dislocation to transmit across the GB (including twin). Based on the criteria, the transmission of incident dislocation is considered more favourable (i) if the angle between the lines of intersection of the incoming and outgoing slip planes with the GB is minimized; (ii) the resolved shear stress of the outgoing slip system is high; and (iii) the interaction minimizes the magnitude of the residual dislocation left at the boundary. It is well known that the first criterion is always satisfied for twin-twin interaction. Nevertheless, the geometrical constraint is not a sufficient parameter to predict the outcome of the interaction. Polcarova et al. [39] point out that the outcome of the twin-twin interaction is largely affected by the local stresses that build up as a result of dislocation-twin interaction, and the source of these local stresses can be credited to the residual dislocation. This is further supported by the atomistic simulations of Cheng et al. [40] who highlight that the local stress field at an atomic level is largely generated by the atomic disarrangements (residual dislocation) in the vicinity of the dislocation incorporation site, and primarily governs the outcomes of the twin-twin interaction process. We therefore validate the need to quantify the twin migration process based on the residual dislocation in this work. The magnitude of $b_{r}$ is an important factor that governs both the process of slip transmission or incorporation along the twin boundary because transmission occurs mainly due to the formation of the slip bands that probably arises from the dissociation of the residual dislocation. As explained before, the magnitude of the sessile residual dislocation gives a precise measurement of the strain incompatibility between the interacting twins. The residual dislocation adequately captures the strain distribution across the twin boundary, and thus proves to be an important parameter to govern the twin migration process.

It can be observed from Equation (6) that the total energy expression incorporates the geometrical parameters such as the length $(d)$ and area $A_{1}$ of the incident twin, the intersecting lengths $\zeta_{1}$ and $\zeta_{2}$, the positions $r_{A}$ and $r_{B}$ of the incident dislocations, and 
the area $A_{2}$ swept by the dislocation segment $b_{2}$ on the verge of twin migration (see Figure 13). The energy contribution due to the length $(d)$ and area $\left(A_{1}\right)$ of the incident twin appears as a constant energy term in the total energy expression (6). Hence, upon minimization with respect to $\zeta_{2}$, they do not contribute to the twin migration stress calculation. As stated earlier, the calculation of the parameters $r_{A}, r_{B}, A_{1}, A_{2}$, and $l_{2}$ is presented in detail in Appendix A2.

\section{Conclusion}

A mesoscale model was developed to determine the magnitude of twin migration stress under twin-twin intersections. The strain distribution and the level of strengthening were largely governed by the residual dislocation. We constructed an energy expression to quantify the level of strengthening as a function of $b_{r}$. The expression allowed for the calculation of twin migration stress upon twin-twin interactions. We did so by minimizing the overall energy expression to determine the applied stress necessary for continued twin boundary progression in the presence of other twin obstacles. The formulation identifies different types of intersections that have been observed experimentally. MD simulation was used in parallel with the experiments to systematically observe the twin migration process at an atomic level and quantify the process in terms of reactions. The modification in the energetics of the twin-twin interaction in the presence of the residual dislocation was obtained via MD and utilized in the total energy expression to calculate the twin migration stress. The predicted twin migration stress conforms to the stress levels observed in experiments. High-resolution DIC measurements facilitated the determination of the strain levels across twin boundaries for different types of twin-twin intersections.

\section{Acknowledgements}

The work was supported by the National Science Foundation, NSF CMMI-[113003]. This support is gratefully acknowledged.

\section{References}

[1] S. Ogata, J. Li and S. Yip, Phys. Rev. B 71 (2005) p.224102.

[2] A.W. Sleeswyk and J.N. Helle, Acta Metall. 9 (1961) p.344.

[3] K. Sumino, Acta Metall. 14 (1966) p.1607.

[4] A.W. Sleeswyk, Acta Metall. 10 (1962) p.705.

[5] A.W. Sleeswyk, Acta Metall. 12 (1964) p.669.

[6] A.W. Sleeswyk and C.A. Verbraak, Acta Metall. 9 (1961) p.917.

[7] J. Levasseur, Mater. Sci. Eng. 4 (1969) p.343.

[8] S. Mahajan, Metall. Trans. A 12 (1981) p.379.

[9] T. Ezaz, M.D. Sangid and H. Sehitoglu, Philos. Mag. 91 (2011) p.1464.

[10] M.D. Sangid, T. Ezaz and H. Sehitoglu, Mater. Sci. Eng., A 542 (2012) p.21.

[11] J.W. Christian and S. Mahajan, Prog. Mater. Sci. 39 (1995) p.1.

[12] L. Patriarca, W. Abuzaid, H. Sehitoglu, H.J. Maier and Y. Chumlyakov, Mater. Charact. 75 (2013) p.165.

[13] B. Edmondson, Proc. R. Soc. A: Math., Phys. Eng. Sci. 264 (1961) p.176.

[14] D. Hull and D.J. Bacon, Introduction to Dislocations, Pergamon Press, Oxford, 1984, p.193. 
[15] C.N. Reid, Metall. Trans. A 12 (1981) p.371.

[16] V. Vítek, Scr. Metall. 4 (1970) p.725.

[17] V. Paidar, Philos. Mag. A 48 (1983) p.231.

[18] M.I. Mendelev, S. Han, D.J. Srolovitz, G.J. Ackland, D.Y. Sun and M. Asta, Philos. Mag. 83 (2003) p.3977.

[19] A. Stukowski, S. Sadigh, P. Erhart and A. Caro, Model. Simul. Mater. Sci. Eng. 7 (2009) p.1.

[20] M.I. Mendelev, S. Han, W.J. Son, G.J. Ackland and D.J. Srolovitz, Phys. Rev. B 76 (2007) p.214105.

[21] M. de Koning, R. Miller, V.V. Bulatov and F.F. Abraham, Philos. Mag. A 82 (2002) p.2511.

[22] A.W. Sleeswyk, Philos. Mag. 8 (1963) p.1467.

[23] D. Hull and D.J. Bacon, Introduction to Dislocations, Vol. 257, Pergamon Press, Oxford, 1984.

[24] J.P. Hirth and J. Lothe, Theory of Dislocations, Wiley, New York, 1982.

[25] J. Harding, Proc. R. Soc. A: Math., Phys. Eng. Sci. 299 (1967) p.464.

[26] J. Harding, Mem. Sci. Rev. Met. 65 (1968) p.245.

[27] H. Suzuki, M. Tanino and K. Aoki, Jpn. J. Appl. Phys. 5 (1966) p.879.

[28] L. Rémy, Acta Metall. 25 (1977) p.711.

[29] J.T. Evans, Scr. Metall. 8 (1974) p.1099.

[30] L.C. Lim, Scr. Metall. 18 (1984) p.1139.

[31] L.C. Lim and R. Raj, Acta Metall. 33 (1985) p.1577.

[32] P.R. Howell and A.P. Jones, J. Mater. Sci. 13 (1978) p.1830.

[33] R. Honda, J. Phys. Soc. Jpn. 16 (1961) p.1309.

[34] H. Jorgens and A. Koechendorfer, Z. Metall. 64 (1973) p.204.

[35] A. Koechendorfer and H. Jorgens, Z. Metall. 62 (1971) p.487.

[36] T. Sakaki and T. Nakamura, J. Iron Steel Inst. Jpn. 59 (1973) p.955.

[37] Z. Shen, R.H. Wagoner and W.A.T. Clark, Acta Metall. 36 (1988) p.3231.

[38] T.C. Lee, I.M. Robertson and H.K. Birnbaum, Metall. Trans. A 21 (1990) p.2437.

[39] M. Polcarova, J. Gemperlova, J. Bradler, A. Jacques, A. George and L. Priester, Philos. Mag. A 78 (1998) p.105.

[40] Y. Cheng, M. Mrovec and P. Gumbsch, Philos. Mag. 88 (2008) p.547.

\section{Appendix A1}

Since twin migration after incorporation occurs by the movement of the $\frac{a}{6}\left[\begin{array}{lll}1 & 1 & 1\end{array}\right]$ dislocation along the twin boundary, a region of atoms ahead of the dislocation successively comes under the influence of the moving dislocation. In the case when the residual dislocation is zero, the energy to be overcome by the moving partial to migrate the twin is represented by the GPFE illustrated in Figure 11. However, when the residual dislocation is present, the energy to be overcome (Figure 12) is elevated as the residual dislocation presents an additional stress field. In the present analysis, we define a region of atoms of area $A$ (defined as the tracing region) on the twin boundary as shown in Figure A1.1 ahead of the moving partial dislocation. We carefully calculate the change in energy over small intervals of time of the tracing region when the partial dislocation passes over it using the following relation:

$$
E_{\mathrm{SF}}=\frac{E_{\text {sheared }}-E_{\text {perfect }}}{A}
$$

where $E_{\text {sheared }}$ is the energy of the crystal corresponding to a normalized displacement $\frac{u_{X}}{b_{p}}\left(u_{X}\right.$ is the displacement of the partial dislocation along the twin boundary) and $E_{\text {perfect }}$ is the perfect lattice energy of the crystal. When the dislocation approaches the tracing region $A$, the energy gradually increases, and reaches to a maximum value when the dislocation overlaps the tracing region 


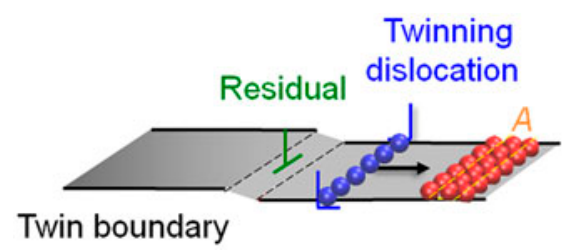

Figure A1.1. (colour online) Schematic showing the process of calculating the modified GPFE. The residual dislocation presents an additional stress field that elevates the $\gamma$-values of the GPFE.

corresponding to $\gamma_{\mathrm{UT}}$. When the dislocation completely passes the tracing region, the energy eventually decreases to a minimum value corresponding to the energy of the perfect lattice. Several authors $[9,10]$ have used a similar approach to calculate the modified energy in the presence of the residual dislocation. We also checked the validity of the method by calculating the GPFE when $b_{r}=0$, and the method yielded an energy profile similar to the one shown in Figure 11.

\section{Appendix A2}

The total energy expression was written earlier as Equation (6). Under the critical resolved shear stress $\tau_{2}$, the outgoing dislocation $b_{2}$ assumes a semicircular segment of radius $\frac{\zeta_{2}}{2}$ as shown in Figure 13. Therefore, the semicircular arc length of the outgoing dislocation is $l_{2}=\frac{\pi \zeta_{2}}{2}$ with a corresponding area of $A_{2}=\frac{\pi \zeta_{2}^{2}}{8}$ in Equation (6). Similarly, on the verge of twin nucleation, the area of the twin $T_{1}$ of constant width $\zeta_{1}$ is $A_{1}=\zeta_{1} r_{A}$.

The initial positions $r_{A}$ and $r_{B}$ of the dislocations of the twin nucleus $T_{1}$ on the verge of twinning has been determined from equilibrium as:

$$
r_{A}=\frac{G b_{1}^{2}}{2 \pi \gamma_{\mathrm{sf}}}, \quad r_{B}=0.732\left\{\frac{G b_{1}^{2}}{2 \pi \gamma_{\mathrm{sf}}}\right\}
$$

We minimize Equation (6) with respect to $\zeta_{1}, \frac{\partial U_{\text {total }}}{\partial \zeta_{1}}=0$, to obtain the following:

$$
\tau_{1}=\frac{-\frac{G b_{1}^{2}}{2 \pi}\left\{\ln \left[\frac{r_{B}-r_{A}}{r_{o}}\right]+\ln \left[\frac{r_{B}}{2 r_{o}}\right]+\ln \left[\frac{r_{A}}{r_{o}}\right]\right\}+\frac{G b_{1}^{2}}{2}+r_{A} \int_{0}^{N_{1}} \gamma_{\text {incidentGPFE }} d \lambda}{b_{1} r_{A}}
$$

Then $\frac{\partial U_{\text {total }}}{\partial \zeta_{2}}=0$, yields

$$
\zeta_{2}=-\frac{\left\{2 \cdot \frac{G b_{r}^{2}}{4 \pi} \ln \left(\frac{R}{w}\right)+\left(\frac{\pi}{2}-1\right) \frac{G b_{2}^{2}}{4 \pi} \ln \left(\frac{R}{w}\right)\right\}}{\frac{\pi}{4} \int_{N_{2}}^{N_{2}+1} \gamma_{\text {modifiedGPFE }} d \lambda-\frac{\pi}{4} \tau_{2} b_{2}}
$$

Using Equations (A2.2) and (5), we obtain:

$$
\tau_{2}=-\frac{\left\{\frac{\pi}{2} \cdot \frac{G b_{2}^{2}}{4 \pi} \ln \left(\frac{R}{w}\right) \cdot \int_{N_{2}}^{N_{2}+1} \gamma_{\text {modifiedGPFE }} d \lambda\right\}}{2 b_{2} \cdot \frac{G b_{r}^{2}}{4 \pi} \ln \left(\frac{R}{w}\right)+(\pi-1) \frac{G b_{2}^{3}}{4 \pi} \ln \left(\frac{R}{w}\right)}
$$

Upon taking the ratio of Equations (A2.3)-(A2.1), the critical stress ratio $\frac{\tau^{M}}{\tau^{M}}$ is obtained as Equation (7). 